\title{
Reconstruction of chaotic signals with application to channel equalization in chaos-based communication systems
}

\author{
Jiuchao Feng ${ }^{1, *, \dagger}$, Chi K. Tse ${ }^{2, \ddagger}$ and Francis C. M. Lau ${ }^{2, \S}$ \\ ${ }^{1}$ Department of Electronic and Information Engineering, South China University of Technology, Guangzhou 510641, China \\ ${ }^{2}$ Department of Electronic and Information Engineering, Hong Kong Polytechnic University, Hong Kong, China
}

\begin{abstract}
SUMMARY
A number of schemes have been proposed for communication using chaos over the past years. Regardless of the exact modulation method used, the transmitted signal must go through a physical channel which undesirably introduces distortion to the signal and adds noise to it. The problem is particularly serious when coherent-based demodulation is used because the necessary process of chaos synchronization is difficult to implement in practice. This paper addresses the channel distortion problem and proposes a technique for channel equalization in chaos-based communication systems. The proposed equalization is realized by a modified recurrent neural network (RNN) incorporating a specific training (equalizing) algorithm. Computer simulations are used to demonstrate the performance of the proposed equalizer in chaos-based communication systems. The Hénon map and Chua's circuit are used to generate chaotic signals. It is shown that the proposed RNN-based equalizer outperforms conventional equalizers as well as those based on feedforward neural networks for noisy, distorted linear and non-linear channels. Copyright (C) 2004 John Wiley \& Sons, Ltd.
\end{abstract}

KEY WORDS: chaos; communications; recurrent neural networks; channel equalization

\section{INTRODUCTION}

Chaotic signals, by virtue of their wideband and deterministic nature, are well suited for carrying information in a spread-spectrum communication environment [1-3]. The wideband feature allows the information to be spread over a wide frequency band, resulting in improved performance in multipath environments and antijamming capability. The deterministic nature allows a high degree of controllability in signal generation and processing. Since 1990s, the use of chaos in communication has been catalysed by the pioneering work of Pecora and Carroll $[4,5]$, who demonstrated the synchronizability of two coupled chaotic systems and hence established the possibility of achieving coherent detection in chaos-based communication. A number of conceptual approaches have been proposed for communication with chaotic signals,

\footnotetext{
*Correspondence to: Jiuchao Feng, Department of Electronic and Information Engineering, South China University of Technology, Guangzhou 510641, China.

${ }^{\dagger}$ E-mail: fengjc@scut.edu.cn

†E-mail: encktse@polyu.edu.hk

${ }^{\S}$ E-mail: encmlau@polyu.edu.hk
} 




Figure 1. Block diagram of part of a chaos-based communication system showing the channel and equalizer.

e.g. chaotic masking [6], chaos shift keying (CSK) [7,8], etc. Most systems proposed and analysed are based on the assumption of a rather ideal communication environment, in which signals are transmitted without distortion and with only a moderate amount of added noise. However, in reality, the performance of a communication system can be seriously impaired by channel effects and noise, especially for coherent-type systems where the unfaithful chaos synchronization can make detection rather unreliable $[9,10]$. Theoretically, if a wideband chaotic signal is transmitted through a band-limited channel, the inevitable loss of spectral components in the received signal may cause the transmitted signal of one symbol to spread over and overlap successive symbol intervals, and this effect is commonly termed inter-symbol interference (ISI) [11]. It has been demonstrated that even simple variations of channel gain or pure phase distortions in the channel may adversely affect the transmitted signal $[12,13]$. In addition to linear distortion, the transmitted signal is subject to other contaminations such as thermal noise, impulse noise and non-linear distortions arising from the modulation process. The idea of channel equalization is to combat the unfavourable channel effects such that the transmitted signal can be preserved with highest integrity [14].

Recently, some approaches have been proposed for combating the effect of channel distortions in chaos-based communication systems. For example, the synchronization-based method [15-17] takes advantage of synchronization between the transmitter and receiver to estimate channel distortion. However, it is not easy to choose a suitable coupling parameter (or adaptive coupling parameter) to ensure that all of the conditional Lyapunov exponents in the demodulator are less than zero so that the receiver can approximately synchronize with the transmitter.

Motivated by the lack of effective channel equalization methods for chaos-based communication systems, the present paper attempts to design a channel equalizer for chaotic signals. Linear and non-linear distortions are considered, in addition to additive white Gaussian noise (AWGN). Specifically, we will employ a modified recurrent neural network (RNN) to realize the equalization task.

This paper is organized as follows. In Section 2, we review the background theory of channel equalization in both chaos-based communication systems and conventional communication systems. Details of the proposed equalizing (learning) algorithm are described in Section 3. The simulation performances of the proposed equalizer are demonstrated in Section 4, where three wellknown channel models (linear and non-linear) will be used to evaluate the performance of the equalizer. Finally, in Section 5, the performance of the proposed equalizer is compared with conventional linear transversal equalizers (LTEs) and those based on feedforward neural networks.

\section{PRELIMINARIES}

Shown in Figure 1 is the block diagram of part of a chaos-based communication system, where $x$ is the transmitted signal produced by the chaotic modulator and $h$ is the transformation 
function of the channel. The output of the channel $s$ is corrupted by noise $\eta$, which is usually modelled as an AWGN process with a zero mean. At the receiver, the received signal, $y$, first goes through an equalizer which cancels the channel effects and estimates the transmitted signal. In this study, we do not consider any specific modulation (demodulation) strategy, and our specific focus here is on cancelling the channel effects.

If the channel $h$ is modelled as a linear operator, the output of the channel, $s$, is simply the convolution of the input sequence $x$ with $h$, i.e. $s=h^{*} x$. Alternatively, $h$ can be modelled as a non-linear operator, which is given generally by $s=h(x)$ [11]. The input to the equalizer is then

$$
y=s+\eta
$$

The problem addressed in this paper may be summarized as follows. Given the noisy and distorted sequence $y$, the problem is to find an equalizer such that the originally transmitted sequence $x$ or at least a delayed and/or phase shifted version of it, can be reconstructed. Therefore, the ideal equalization requires $\hat{x}=\delta_{t L} \mathrm{e}^{\mathrm{j} \theta} x$ be achieved, where $t$ is time instant, $L$ is a time delay, $\theta$ is a constant phase shift, and $\delta$ is the Kronecker delta function.

\subsection{Conventional equalizers}

The problem of equalization can be interpreted as one of inverse modelling [18], i.e. deconvolving the received sequence in order to reconstruct the original message. The conventional equalizer architecture is shown in Figure 2. The received training sequence, $\{y(t)\}$, is filtered by an LTE which produces an output for the sample at time $t-L$ based on $m$ most recent channel observations made at time $t$, where the integers $m$ and $L$ are the equalizer's order (tap) and delay, respectively. In the training stage (Figure 2(a)), some preset sample signals are transmitted via the channel, and the received samples are used to train the LTE. Usually, a stochastic gradient algorithm, such as the least mean square (LMS) algorithm [19], is used to adjust the tap weights $w_{i}, i=0,1,2, \ldots, m-1$, in the light of the error signal $e(t-L)$, which is given by

$$
e(t-L)=d(t-L)-\hat{x}(t-L)
$$

where $d(t-L)$ is the desired signal at time $t-L$, and $\hat{x}(t-L)$ is the estimate of $x(t-L)$. In the equalizing stage (Figure 2(b)), the trained LTE gives the estimate of $x(t-L)$ from the unknown received sequence when communication commences.


Figure 2. LTE used in (a) training stage, and (b) equalizing stage. 


\section{2. $R N N$ and equalization}

The aforementioned equalization problem can be regarded as a non-linear modelling problem. The non-linear auto-regressive moving average model (NARMA), which is a widely used tool for modelling non-linear dynamical system (e.g. in time series processing, non-linear signal reconstruction, etc.), can be used to describe the aforementioned system [20]. Typically, we write

$$
x(t)=\varpi(y(t-1), y(t-2), \ldots, y(t-M), e(t-1), e(t-2), \ldots, e(t-N))+e(t)
$$

where $e(t)$ is the error signal at time instant $t$ between the original and the estimated signal, $\varpi$ is an unknown function, $M$ and $N$ are the time delays of the input signal and the error signal, respectively. The conditional mean of $x$ based on the infinite past observations is

$$
\begin{aligned}
\hat{x}(t)= & E[\varpi(y(t-1), y(t-2), \ldots, y(t-M), e(t-1), \\
& e(t-2), \ldots, e(t-N)) \mid y(t-1), y(t-2), \ldots]
\end{aligned}
$$

where $E$ denotes expectation. Suppose that the NARMA model is invertible in the sense that there exists a function $\psi$ such that

$$
x(t)=\psi(y(t-1), y(t-2), \ldots)+e(t)
$$

Then, given the infinite past observations $y(t-1), y(t-2), \ldots$, one can in principle use the above equation to estimate $e(t-j)$ in (3). In this case the conditional mean estimate is

$$
\hat{x}(t)=\boldsymbol{\varpi}(y(t-1), y(t-2), \ldots, y(t-M), e(t-1), e(t-2), \ldots, e(t-N))
$$

Since in practice only a finite observation record is available, one cannot perform computation using (6). However, it is possible to approximate (6) by the recursive algorithm [20]

$$
\hat{x}(t)=\varpi(y(t-1), y(t-2), \ldots, y(t-M), \hat{e}(t-1), \hat{e}(t-2), \ldots, \hat{e}(t-N))
$$

where $\hat{e}(j)=x(j)-\hat{x}(j), j=t-1, t-2, \ldots, t-N$. The above equation can be approximated by the following recurrent model [20-22]:

$$
\hat{x}(t)=\sum_{i=1}^{N} u_{i} \varphi\left(\sum_{j=1}^{M} \breve{w}_{i j} y(t-j)+\sum_{j=1}^{N} \breve{w}_{i j}^{\prime}(x(t-j)-\hat{x}(t-j))+\theta_{i}\right)
$$

which is actually a special case of a general RNN to be described in the following. Here, $u_{i}, \breve{w}_{i j}$ and $\breve{w}_{i j}^{\prime}$ are coefficients. $\theta_{i}$ is a parameter and $\varphi$ is a non-linear function.

An RNN is shown in Figure 3, which is a three-layer network consisting of the input layer, the hidden layer (processing layer) and the output layer. The input vector of the input layer at time instant $t$ is $v(t)$, which is defined as

$$
v(t)=\left[v_{1}(t), v_{2}(t), \ldots, v_{M}(t), v_{M+1}(t), \ldots, v_{M+N+1}(t)\right]^{\mathrm{T}}
$$

where $v_{i}(t), 2 \leqslant i \leqslant M+1$, is the external input which is the delayed version of $y$, i.e. $v_{i}(t)=y$ $(t-(i-1))$, and $v_{i}, M+2 \leqslant i \leqslant M+N+1$, is the feedback input of the $i$ th input unit at time instant $t$. Also, $N$ is the number of hidden layer units and $v_{1}$ is the bias input which has been fixed at " $+1 "$ in this paper.

The internal activity of the $i$ th hidden unit at time instant $t$ is given by

$$
r_{i}(t)=\sum_{j=1}^{M+N+1} w_{i j}(t) v_{j}(t)
$$




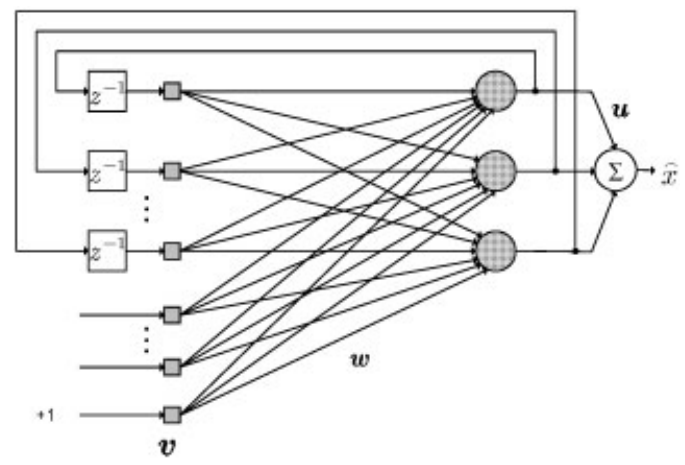

Figure 3. Block diagram of a modified RNN.

where $w_{i j}(t)$ is the connection weight between the $i$ th hidden unit and the $j$ th input unit at time instant $t$. At the next time step $t+1$, the output of the $i$ th neuron, $q_{i}(t+1)$, is computed using a non-linear activation function, $\varphi(\cdot)$, yielding

$$
q_{i}(t+1)=\varphi\left(r_{i}(t)\right)
$$

In this study, we choose

$$
\varphi(x)=\tanh (c x)
$$

where $c$ is constant. Let $u_{i}(t)$ be the connection weight between the $i$ th hidden unit and the output unit. The output of the output unit is given by

$$
\hat{x}(t+1)=\sum_{i=1}^{N}\left[u_{i}(t) q_{i}(t+1)\right]
$$

For $1 \leqslant i \leqslant N$, and letting

$$
w_{i j}=-\breve{w}_{i j} u_{i} \quad \text { for } \quad M+2 \leqslant j \leqslant M+N+1
$$

and

$$
w_{i j}(\text { if } M>N)= \begin{cases}\breve{w}_{i j}+\breve{w}_{i j}^{\prime} & \text { for } 2 \leqslant j \leqslant N+1 \\ \breve{w}_{i j} & \text { for } N+2 \leqslant j \leqslant M+1\end{cases}
$$

we can easily see that (8) is a special case of (13) for $M>N$. Similarly, for the cases where $M<N$ and $M=N$, the same conclusion holds.

The above estimation procedures, together with the training algorithm described in the following section, can be used to realize the equalization task.

\section{TRAINING ALGORITHM}

Let $d(t+1)$ be the desired output of the output unit at time instant $t+1$. The error signal $e(t+1)$ is

$$
e(t+1)=d(t+1)-\hat{x}(t+1)
$$


The weight between the hidden layer and the output unit is then updated by an LMS algorithm [19], i.e.

$$
u_{i}(t+1)=u_{i}(t)+\beta_{1} e(t+1) q_{i}(t+1)
$$

where $\beta_{1}$ is the learning rate. The instantaneous sum of squared errors of the network is defined as $\varepsilon^{0}(t+1)=\frac{1}{2} e^{2}(t+1)$ (here, there is only one output unit, see Figure 3 ). Also, we define the local gradient of the $i$ th hidden unit at time instant $t+1, \gamma_{i}(t+1)$, as

$$
\begin{aligned}
\gamma_{i}(t+1) & =-\frac{\partial \varepsilon^{0}(t+1)}{\partial r_{i}(t)} \\
& =e(t+1) u_{i}(t) \frac{\partial q_{i}(t+1)}{\partial r_{i}(t)} \\
& =e(t+1) u_{i}(t) \varphi^{\prime}\left(r_{i}(t)\right)
\end{aligned}
$$

where $\varphi^{\prime}(\cdot)$ is the derivative of $\varphi$ with respect to its argument. According to the delta learning law, the weight $w_{i j}(i=1,2, \ldots, N, j=1,2, \ldots, M+N+1)$ can be updated as follows:

$$
w_{i j}^{-}(t+1)=w_{i j}(t)+\beta_{2} \gamma_{i}(t+1) v_{j}(t)
$$

where $\beta_{2}$ is the learning rate. Now, define the instantaneous sum of squared errors for the hidden layer units as

$$
\varepsilon(t)=\frac{1}{2} \sum_{k=1}^{N} e_{k}^{2}(t)
$$

where $e_{k}(t)$ is the difference (error) in the output of the $k$ th hidden unit before and after the weight $w_{i j}$ is updated. Then, the instantaneous weight is updated as

$$
w_{i j}(t+1)=w_{i j}^{-}(t+1)-\beta_{3} \frac{\partial \varepsilon(t)}{\partial w_{i j}^{-}(t+1)}
$$

where $\beta_{3}$ is learning rate. From (11) and (20), we have

$$
\begin{aligned}
\frac{\partial \varepsilon(t)}{\partial w_{i j}^{-}(t+1)} & =\sum_{k=1}^{N} e_{k}(t) \frac{\partial e_{k}(t)}{\partial w_{i j}^{-}(t+1)} \\
& =-\sum_{k=1}^{N} e_{k}(t) \frac{\partial q_{k}^{+}(t+1)}{\partial w_{i j}^{-}(t+1)}
\end{aligned}
$$

where $q_{k}^{+}(t+1)$ is the output of the $k$ th hidden unit after the weight $w_{i j}$ is updated to $w_{i j}^{-}(t+1)$. To determine the partial derivative $\partial q_{k}^{+}(t+1) / \partial w_{i j}^{-}(t+1)$, we differentiate (10) and (11) with respect to $w_{i j}$. By applying the chain rule, we obtain

$$
\begin{aligned}
\frac{\partial q_{k}^{+}(t+1)}{\partial w_{i j}^{-}(t+1)} & =\frac{\partial q_{k}^{+}(t+1)}{\partial r_{k}^{-}(t+1)} \frac{\partial r_{k}^{-}(t+1)}{\partial w_{i j}^{-}(t+1)} \\
& =\varphi^{\prime}\left(r_{k}^{-}(t+1)\right) \frac{\partial r_{k}^{-}(t+1)}{\partial w_{i j}^{-}(t+1)}
\end{aligned}
$$


where $r_{k}^{-}(t+1)$ corresponds to the updated internal state of hidden unit $k$. By using (11), we get

$$
\begin{aligned}
\frac{\partial r_{k}^{-}(t+1)}{\partial w_{i j}^{-}(t+1)} & =\sum_{n=1}^{M+N+1} \frac{\partial}{\partial w_{i j}^{-}(t+1)}\left[w_{k n}^{-}(t+1) v_{n}(t)\right] \\
& =\sum_{n=1}^{M+N+1}\left[w_{k n}^{-}(t+1) \frac{\partial v_{n}(t)}{\partial w_{i j}^{-}(t+1)}+v_{n}(t) \frac{\partial w_{k n}^{-}(t+1)}{\partial w_{i j}^{-}(t+1)}\right]
\end{aligned}
$$

Note that the derivative $\partial w_{k n}^{-}(t+1) / \partial w_{i j}^{-}(t+1)$ is equal to one if $k=i$ and $n=j$, and is zero otherwise. Thus, we may rewrite (24) as

$$
\frac{\partial r_{k}^{-}(t+1)}{\partial w_{i j}^{-}(t+1)}=\sum_{n=1}^{M+N+1}\left[w_{k n}^{-}(t+1) \frac{\partial v_{n}(t)}{\partial w_{i j}^{-}(t+1)}\right]+\delta_{i k} v_{j}(t)
$$

From (9), we have

$$
\frac{\partial v_{n}(t)}{\partial w_{i j}^{-}(t+1)}= \begin{cases}\frac{\partial q_{n}^{+}(t)}{\partial w_{i j}^{-}(t+1)} & \text { for } n=M+2, \ldots, M+N+1 \\ 0 & \text { otherwise }\end{cases}
$$

We may then combine (23), (25) and (26) to yield

$$
\frac{\partial q_{k}^{+}(t+1)}{\partial w_{i j}^{-}(t+1)}=\varphi^{\prime}\left(r_{k}^{-}(t+1)\right)\left[\sum_{n=M+2}^{N+M+1} w_{k n}^{-}(t+1) \frac{\partial q_{n}^{+}(t)}{\partial w_{i j}^{-}(t+1)}+\delta_{i k} v_{j}(n)\right]
$$

where $\delta$ is the Kronecker delta function. We now define a dynamical system described by a triply indexed set of variables $\left\{\Omega_{i j}^{n}(t+1)\right\}$, where

$$
\Omega_{i j}^{n}(t+1)=\frac{\partial q_{n}^{+}(t)}{\partial w_{i j}^{-}(t+1)}
$$

for $j=1,2, \ldots, M+N+1, i=1,2, \ldots, N$, and $n=M+2, \ldots, M+N+1$. For each time step $t$ and all appropriate $n, i$ and $j$, the dynamics of the system so defined is governed by

$$
\Omega_{i j}^{k}(t+1)=\varphi^{\prime}\left(r_{k}^{-}(t+1)\right)\left[\sum_{n=M+2}^{M+N+1} w_{k n}^{-}(t+1) \Omega_{i j}^{n}(t+1)+\delta_{i k} v_{j}(t)\right]
$$

Finally, the weight between the input layer and the hidden layer is updated by

$$
w_{i j}(t+1)=w_{i j}^{-}(t+1)+\beta_{3} \sum_{k=1}^{N} e_{k}(t+1) \Omega_{i j}^{k}(t+1)
$$

The above procedure is repeatedly applied to all input sample pairs during the training stage.

\section{SIMULATION STUDY}

In this section we simulate a chaos-based communication system which is subject to channel distortion and AWGN. Our purpose is to test the ability of the proposed equalizer in combating the channel effects and noise. 


\subsection{Chaotic signal transmission}

Two chaotic systems will be used to evaluate the performance of the proposed equalizer in this paper. The first system is based on the Hénon map:

$$
\begin{aligned}
& x_{1}(t+1)=1-\alpha_{1} x_{1}^{2}(t)+x_{2}(t) \\
& x_{2}(t+1)=\alpha_{2} x_{1}(t)
\end{aligned}
$$

where $\alpha_{1}$ and $\alpha_{2}$ are the bifurcation parameters fixed at 1.4 and 0.3 , respectively. In particular, we select $x_{2}$ as the transmitted signal, which guarantees that the dynamics can be reconstructed by using the observed distorted samples of the transmitted signal [23].

The second system is based on the Chua's circuit [24], which is given by the following dimensionless equations:

$$
\begin{aligned}
& \dot{x}_{1}=\alpha_{3}\left(x_{2}-\kappa\left(x_{1}\right)\right) \\
& \dot{x}_{2}=x_{1}-x_{2}+x_{3} \\
& \dot{x}_{3}=\alpha_{4} x_{2}
\end{aligned}
$$

where $\kappa(\cdot)$ is a piecewise-linear function given by

$$
\kappa\left(x_{1}\right)= \begin{cases}m_{1} x_{1}+\left(m_{0}-m_{1}\right) & \text { for } x_{1} \geqslant 1 \\ m_{0} x_{1} & \text { for }\left|x_{1}\right|<1 \\ m_{1} x_{1}-\left(m_{0}-m_{1}\right) & \text { for } x_{1} \leqslant-1\end{cases}
$$

with $m_{0}=-\frac{1}{7}$ and $m_{1}=\frac{2}{7}$. For different values of $\alpha_{3}$ and $\alpha_{4}$, the system operates in different regimes, e.g. periodic and chaotic regimes. The well-known double scroll Chua's attractor, for example, is obtained for $\alpha_{3}=9$ and $\alpha_{4}=-\frac{100}{7}$. The attractor has the largest Lyapunov exponent and the Lyapunov dimension equal to 0.23 and 2.13, respectively [25]. In this case, we select $x_{3}$ as the transmitted signal. This choice of the transmitted signal guarantees that the dynamics of the transmission system can be reconstructed by using the observed distorted samples of the transmitted signal [23].

\subsection{Filtering effect of communication channels}

Three channel models will be used to test the performance of the proposed equalizer in this paper. The first two channels are linear channels which can be described in the $z$ domain by the following transformation functions:

$$
\begin{gathered}
H_{1}(z)=1+0.5 z^{-1}(\text { Channel I) } \\
H_{2}(z)=0.3+0.5 z^{-1}+0.3 z^{-2} \text { (Channel II) }
\end{gathered}
$$

These two channel models are widely used to evaluate the performance of equalizers in communication systems [26]. Let us consider the frequency responses of the channels. The amplitude- and phase-frequency responses of the channels are shown in Figure 4. It is worth noting that from Figure 4(a), Channel II has a deep spectrum null at a normalized angular frequency of 2.56, which is difficult to equalize by the usual LTE [11].

The third channel to be studied is a non-linear channel, which is shown in Figure 5. This channel is also widely used for testing the performance of equalizers [27]. The model can be 

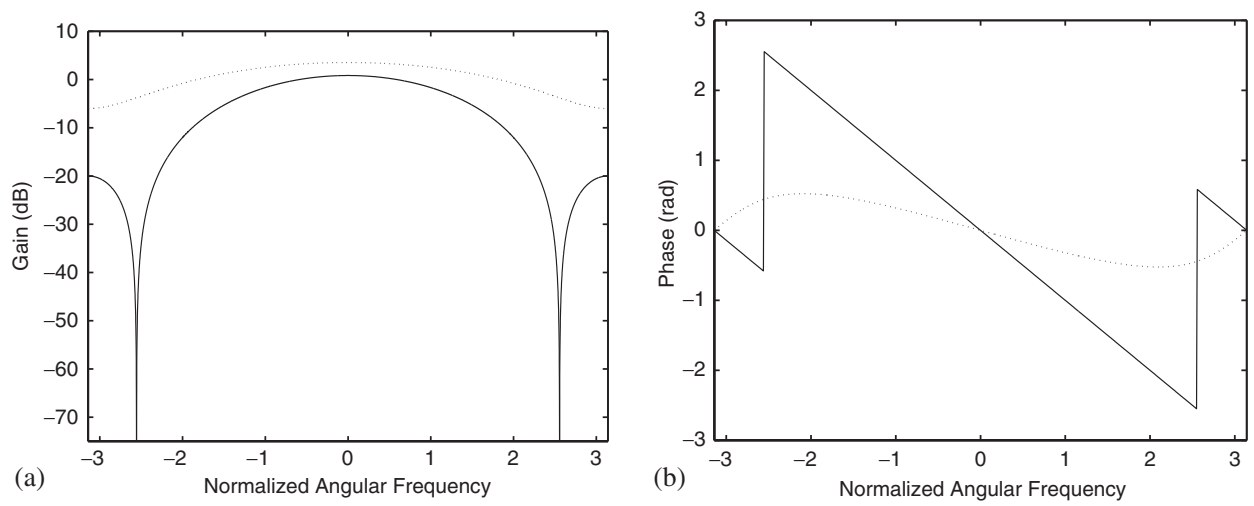

Figure 4. Frequency responses of Channel I (dotted line)and Channel II (solid line) according to (34) and (35). (a) Magnitude responses; (b) phase responses.

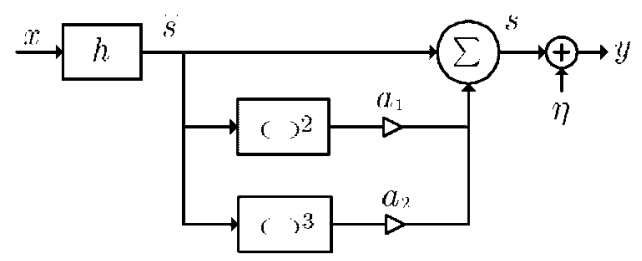

Figure 5. Non-linear channel model studied in this paper.

described by

$$
y=\breve{s}+a_{1} \breve{s}^{2}+a_{2} \breve{s}^{3}+\eta
$$

where $a_{1}$ and $a_{2}$ are channel parameters which are fixed at 0.2 and -0.1 , respectively, and $\breve{s}$ is the output of the linear part of the channel which is given by

$$
\breve{s}(t)=0.3482 x(t)+0.8704 x(t-1)+0.3482 x(t-2)
$$

Thus, the transformation function of the linear component can be expressed as

$$
H(z)=0.3482+0.8704 z^{-1}+0.3482 z^{-2}
$$

As an example to illustrate the channel effects, we consider a communication event, in which the transmitted signal $x_{2}$ generated from (31) passes through Channel I. When the signal-tonoise ratio (SNR) is $10 \mathrm{~dB}$, the FFT spectra of the transmitted signal and the received signal are shown in Figures 6(a) and 6(b), from which we clearly observe the wideband property of the transmitted signal and the distortion caused by the channel. Furthermore, the return maps reconstructed from the transmitted signal, the distorted signal due to Channel I, and the noisy received signal are shown in Figure 7. It has been shown [13] that without an equalizer, the simple inverse system approach will give unacceptable performance even when the channel, besides AWGN, is an ideal allpass filter, i.e. $h=1$. 

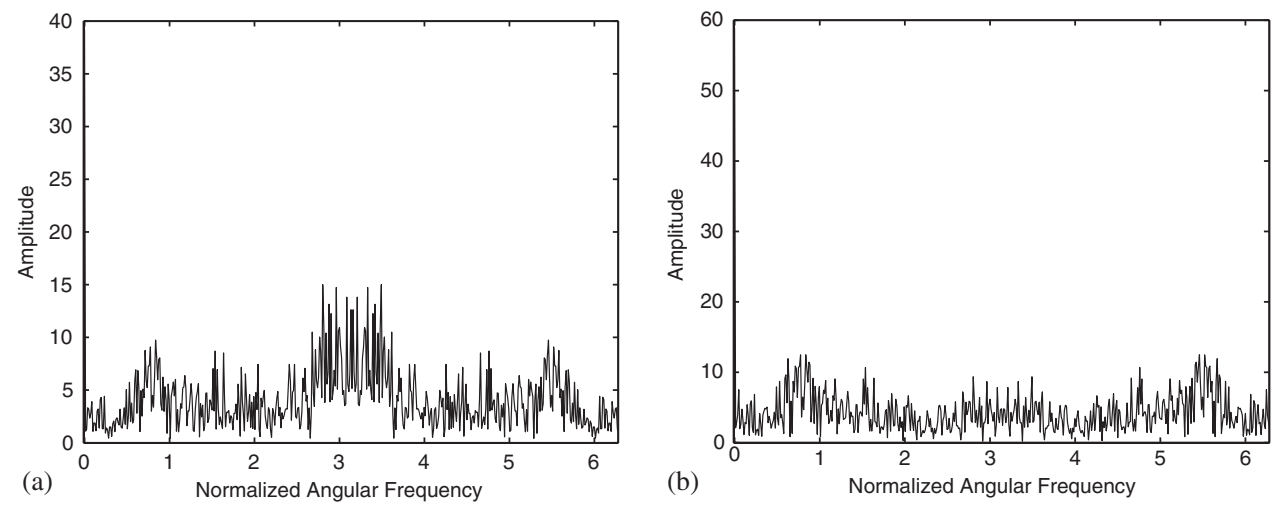

Figure 6. Illustration of channel effects. FFT amplitude spectrum of signal versus normalized angular frequency for (a) transmitted signal $x_{2}$ from (31), and (b) received signal $y$ after passing through Channel I and contaminated by noise at $\mathrm{SNR}=10 \mathrm{~dB}$.
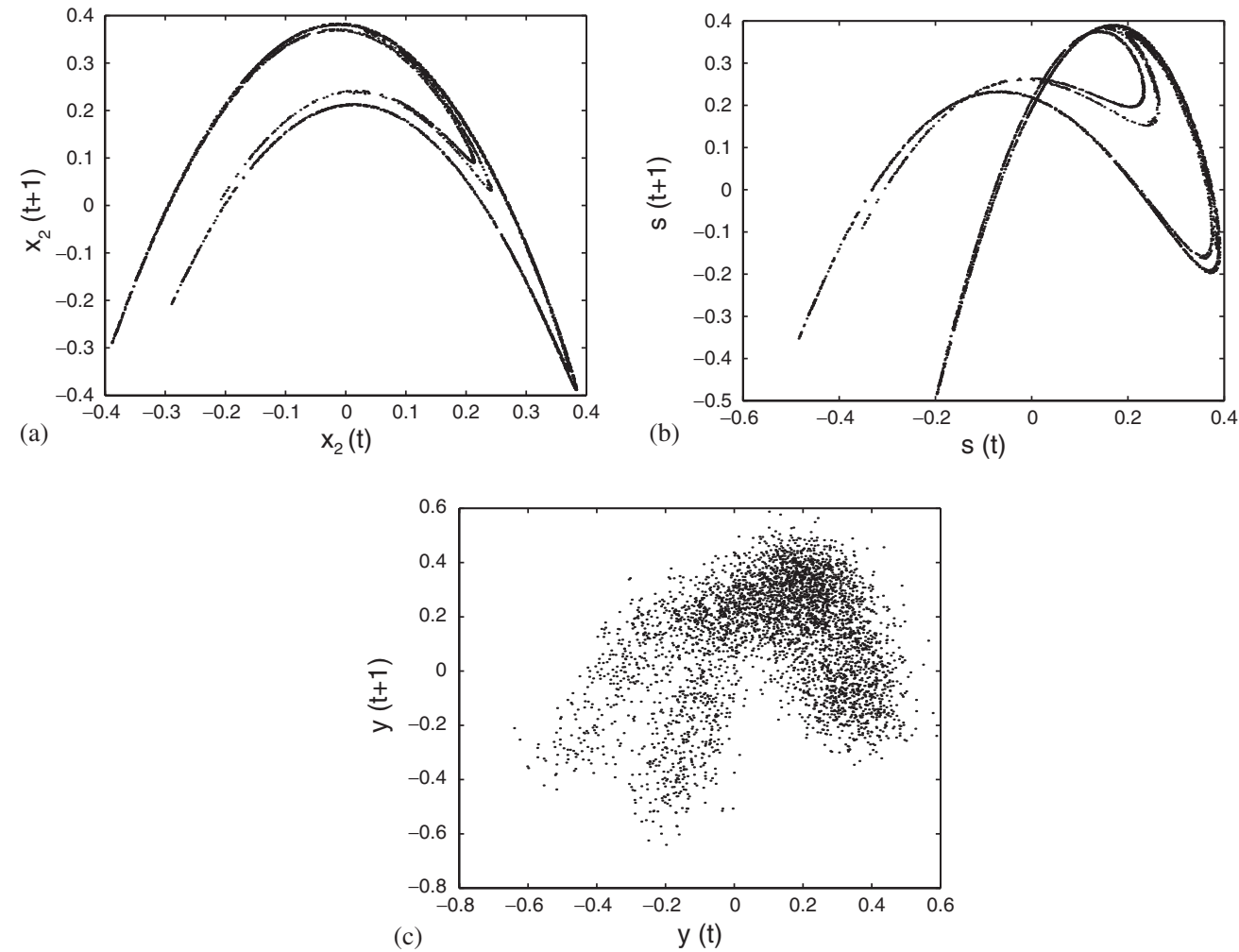

Figure 7. Illustration of channel effects. Return maps reconstructed from (a) the transmitted $x_{2}$ from (31), (b) the distorted $s(t)$, and (c) the received signal $y$ after passing through Channel I and contaminated by noise at SNR $=10 \mathrm{~dB}$. 


\subsection{Results}

The equalization for each channel model consists of two stages. The first is the training or adaptation stage in which the equalizer makes use of some partially known sampling pairs to adapt to the communication environment. When the training or adaptation is completed, actual communication commences.

In the training stage, 600 training sample sets are used, each having seven transmitted signals and one known signal at the receiver. Also, noise is added to the training samples at an SNR of $10 \mathrm{~dB}$ during the training stage. In the simulation, the RNN is assigned with $M=7$, which is subject to the requirement for the embedding dimension in the Takens' theory [28], and $N=6$. The training of the equalizer completes when the mean-square-error (MSE) for all samples is less than $10^{-7}$. In this paper, we define the MSE as $\left\langle(\hat{x}-x)^{2}\right\rangle$, where " \langle\rangle " is an averaging operator. Results are summarized as follows:

(1) When the RNN-based equalizer is applied to equalize Channels I and II, the following results are obtained:

- For the Hénon-map system, Figure 8 shows the MSE of the equalized signals versus the number of iterations in the training duration for Channels I and II. The MSE for each channel in the training stage is averaged over 40 independent realizations in this study. It can be seen from Figure 8 that the equalizer completes the training in approximately 500 iterations for Channel I and 600 iterations for Channel II. In this paper, one iteration means that the equalizer is trained once with all training samples. The trained equalizers are then used to test their performances when actual communication takes place. Figure 9 shows the MSE versus SNR for Channels I and II. When the SNR exceeds $14 \mathrm{~dB}$, the MSE of the equalized signal for the two channels is less than $-80 \mathrm{~dB}$.



Figure 8. MSE of the equalized samples, averaged over 40 independent realizations, versus the number of iterations for the Hénon-map system for Channel I (the solid line) and Channel II (dotted line). 


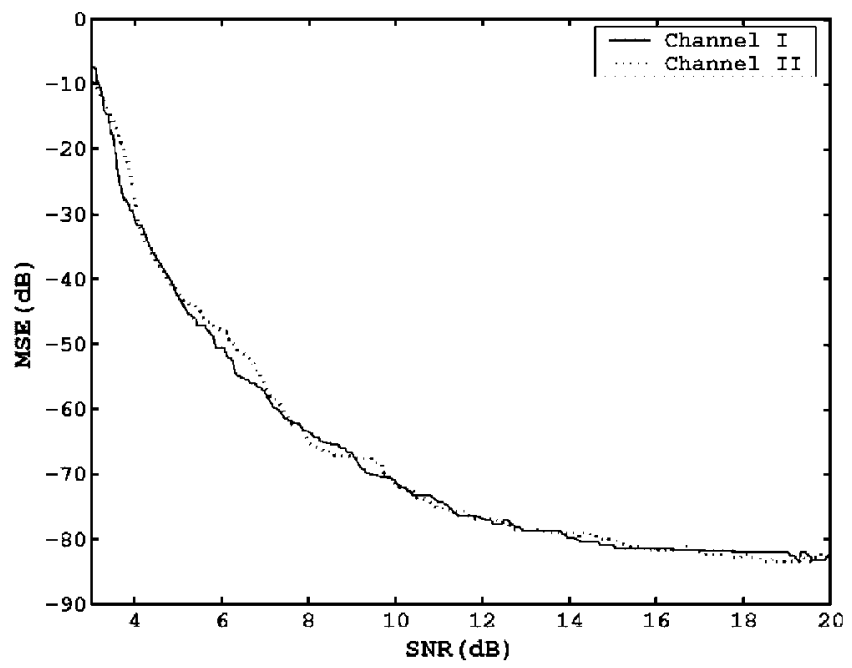

Figure 9. MSE performance of the recovered $\hat{x}_{2}$ of the Hénon-map system versus SNR for Channel I (solid line) and Channel II (dotted line).

- For the Chua's system, it is found that the equalizer completes the training after approximately 500 iterations for Channel I and 700 iterations for Channel II. The trained equalizers are then used to test their performances when actual communication takes place. We find that the MSE decreases with the increased SNR, and when SNR is more than $14 \mathrm{~dB}$, MSE of the equalized signal for the two channels is less than $-76.3 \mathrm{~dB}$.

(2) When the RNN-based equalizer is applied to equalize the non-linear channel described in Section 4.2, the following results are obtained:

- For the Hénon-map system, it is found that the equalizer completes its training after about 800 iterations in the training stage, and the MSE decreases with the increased SNR in the equalizing stage. When SNR is equal to $14 \mathrm{~dB}$ in the equalizing phase, the MSE is $-73.8 \mathrm{~dB}$.

- For the Chua's system, similarly, it is found that the equalizer completes its training after about 900 iterations, and the MSE decreases with the increased SNR in the equalizing stage. When the SNR is equal to $14 \mathrm{~dB}$ in the equalizing stage, the MSE of the equalized signal is approximately $-76 \mathrm{~dB}$.

\section{COMPARISONS AND DISCUSSIONS}

Firstly, we compare the proposed RNN-based equalizer with conventional LTEs. In our study, 13- and 15-tap LTEs (see Section 2.1) are applied to equalize Channels I and II. The results are summarized as follows. Figures 10(a) and 10(b) show the MSE performance of the equalized signal versus the SNR of the channel for the communication systems based on the Hénon map and the Chua's circuit, respectively. When the SNR is $14 \mathrm{~dB}$, the MSE is -19.2 and $-16.7 \mathrm{~dB}$ for Channels I and II, respectively, for the Hénon-map system. Here, the RNN-based equalizer 

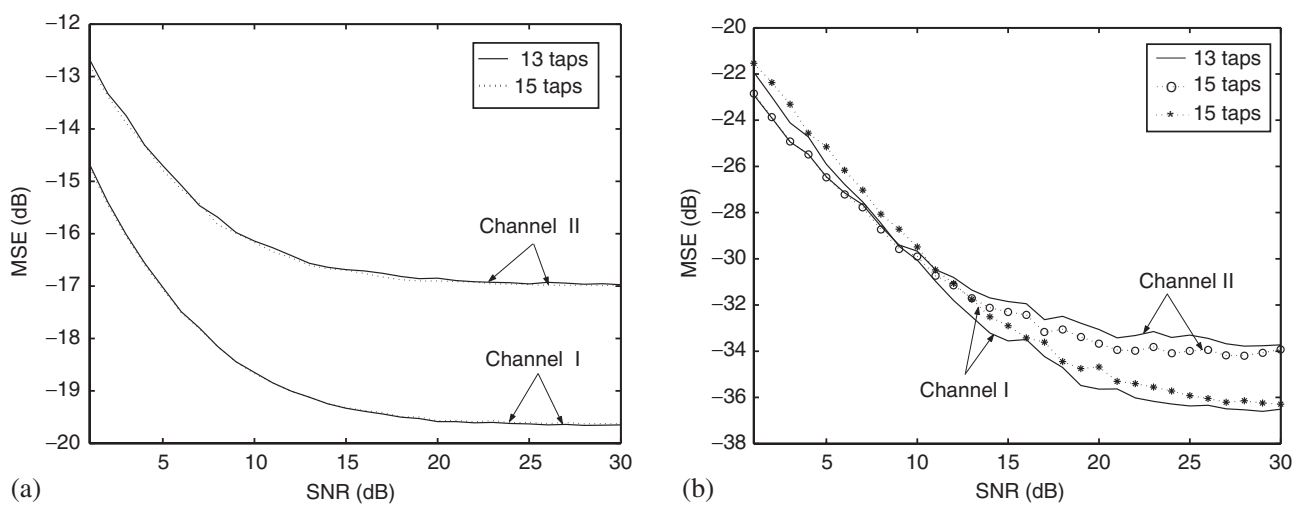

Figure 10. MSE performance of LTEs versus SNR for (a) Hénon-map, and (b) Chua's system. 13-tap equalizer (solid line) and 15-tap equalizer (dotted line).

outperforms these LTEs by 60 and $63.3 \mathrm{~dB}$ for Channels I and II, respectively. Likewise, for the Chua's system, the MSE is -32.8 and $-31.8 \mathrm{~dB}$ for Channels I and II, respectively. In this case, the RNN-based equalizer outperforms the LTEs by 45.5 and $44.5 \mathrm{~dB}$ for Channels I and II, respectively. Also, the LTEs are found completely inadequate for equalizing the non-linear channel, and results are omitted here.

Secondly, we discuss the performance of the proposed equalizer based on the modified RNN in this paper. As described in Section 2.2 the equalization task can be modelled as a general RNN. Basically, artificial neural networks (ANN) can perform complex mapping between its input and output space. Specifically, unlike static networks, for example, feedforward neural networks, RNN can perform temporally extended tasks, for which static networks have serious limitations [29]. The salient property of the RNN is that the outputs of the hidden units are fed back at every time step to provide an additional input. This recurrence enables the filtered data of the previous period to be used as an additional input in the current period. In other words, in each time period the network is subject to not only the new noisy inputs data but also the past history of all noisy inputs as well as their filtered counterparts. This additional information of filtered input history acts as an additional guidance to evaluate the current noisy input and its signal component. Therefore, RNN provides a suitable platform to realize the above equalization task. Here, as an example, we use a feedforward neural network without recurrent input, which is a special case of the one shown in Figure 3 (i.e. $N=0$ in the input layer), to realize the same equalization task for Channels I and II. In the adaption stage, the back propagation algorithm [30] is used to train the equalizer. Figure 11 shows the MSE performance of the equalized signal versus the SNR of the channel. We can see by comparing Figures 9 and 11 that when SNR is $14 \mathrm{~dB}$, the RNN-based equalizer outperforms the static feedforward network by 48.9 and $51.8 \mathrm{~dB}$ for Channels I and II, respectively.

\section{CONCLUSIONS}

Channel equalization in chaos-based communication systems has been studied in this paper. The main focus is the kind of channel distortion arising from linear delays as well as non- 


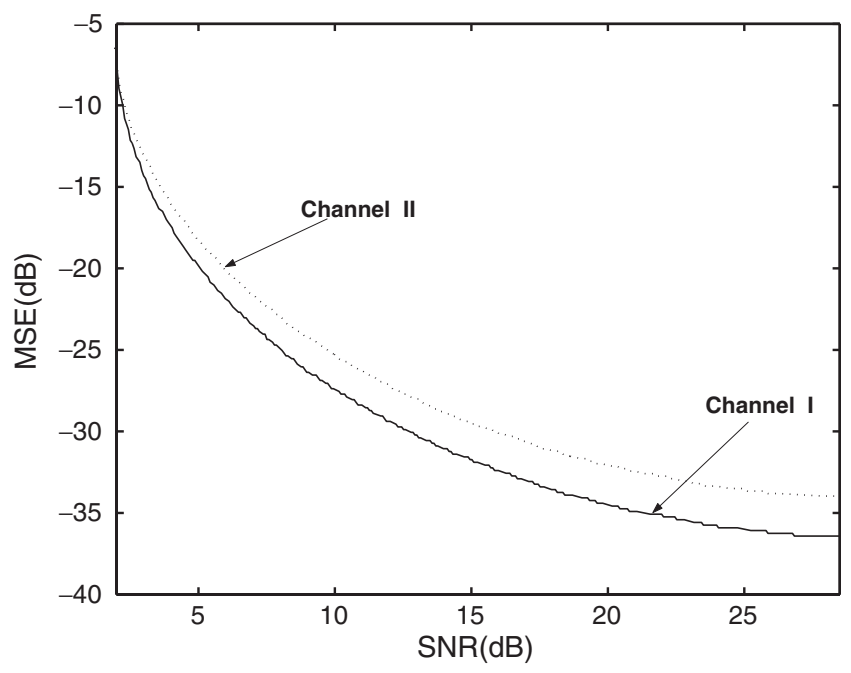

Figure 11. The equalization performance by using an equalizer based on the feedforward neural network without recurrency using the back propagation learning algorithm, in which the Hénon-map is employed in the transmitter.

linearity. The aim is to derive an effective equalization method such that a transmitted chaotic signal can be preserved with minimal distortion as it passes through a communication channel. Our design approach is based on the use of a recurrent neural network, which has the memory ability to combat dynamical changes, for "probing" the channel characteristics. The equalizer essentially consists of a modified recurrent neural network which incorporates a specific learning algorithm. The Hénon-map system and Chua's system have been used as chaos generating systems in the simulation study, and three typical linear and non-linear channels are considered. It has been found that the proposed equalizer can effectively "undo" the channel effects, permitting the chaotic signal to be reconstructed at the receiver. It should be noted that in present work, the equalizer has only been tested for some specific linear and non-linear channel models, which represent basic types of channel distortions. However, in practical wireless communication channels, the types of distortion may arise from a wider range of non-linear phenomena (e.g. fading channels), which can make the problem much harder to tackle. We believe that future research will therefore focus on further theories and techniques for tracking and modelling these channels.

\section{REFERENCES}

1. Heidari-bateni G, McGillem CD. A chaotic direct-sequence spread-spectrum communication system. IEEE Transactions on Communications 1994; 42:1524-1527.

2. Parlitz U, Chua LO, Kocarev L, Hale KS, Shang A. Transmission of digital signals by chaotic synchronization. International Journal of Bifurcation and Chaos 1992; 2:973-977.

3. Yang T, Chua LO. Chaotic digital code-division multiple access (CDMA) communication systems. International Journal of Bifurcation and Chaos 1997; 7:2789-2806.

4. Pecora LM, Carroll TL. Synchronization in chaotic systems. Physical Review Letters 1990; 64:821-824.

5. Pecora LM, Carroll TL. Driving systems with chaotic signals. Physical Review A 1991; 44:2374-2383. 
6. Kocarev L, Halle KS, Eckert KS, Chua LO, Parlitz U. Experimental demonstration of secure communications via chaotic synchronization. International Journal of Bifurcation and Chaos 1992; 2:709-713.

7. Dedieu H, Kennedy MP, Hasler M. Chaos shift keying: modulation and demodulation of a chaotic carrier using self-synchronizing Chua's circuits. IEEE Transactions on Circuits and Systems II 1993; 40:634-642.

8. Chow TWS, Feng JC, Ng KT. Chaotic network synchronization with application to communications. International Journal of Communication Systems 2001; 14:217-230.

9. Johnson GA, Mar DJ, Carroll TL, Pecora LM. Synchronization and parameter tracking in chaotic systems. In Proceedings of the Fourth Experimental Chaotic Conference. World Scientific: Singapore, 1998.

10. Kisel A, Dedieu H, Schimming T. Maximum likelihood approaches for noncoherent communications with chaotic carriers. IEEE Transactions on Circuits and Systems I 2001; 48:533-542.

11. Proakis JG. Digital Communications (3rd edn). McGraw-Hill: New York, 1995.

12. Oppenheim AV, Cuomo KM, Baron RJ, Fredman AE. Channel equalization for communication with chaotic signals. In Chaotic, Fractal and Nonlinear Signal Processing, Katz RA (ed.), AIP Press: New York, 1996.

13. Feng JC, Tse CK. On-line adaptive chaotic demodulator based on radial-basis-function neural networks. Physical Review E 2001; 63:026202:1:10.

14. Ciftci M, Williams DB. A novel channel equalizer for chaotic digital communications systems. Proceedings of IEEE ICASSP 1999; 3:1301-1304.

15. Cuomo KM, Oppenheim AV, Barron RJ. Channel equalization for self-synchronizing chaotic systems. Proceedings of IEEE ICASSP 1996; 3:1605-1608.

16. Sharma N, Ott E. Combating channel distortions in communication with chaotic systems. Physical Letters A 1998; 248:347-352.

17. Chua LO, Yang T, Zhong GQ, Wu CW. Synchronization of Chua's circuits with time-varying channels and parameters. IEEE Transactions on Circuits and Systems I 1996; 43:862-868.

18. Qureshi SUH. Adaptive equalization. Proceedings of IEEE 1985; 73:1349-1387.

19. Haykin S. Adaptive Filter Theory (3rd edn). Prentice-Hall Inc.: Englewood Cliffs, NJ, 1996.

20. Box GE, Jenkins GM. Time Series Analysis: Forecasting and Control (3rd edn), Prentice-Hall Inc.: Englewood Cliffs, NJ, 1994.

21. Williams RJ, Zipser D. A learning algorithm for continually running fully recurrent neural networks. Neural Computation 1989; 1:270-280.

22. Connor JT, Martin RD, Atlas LE. Recurrent neural networks and robust time series prediction. IEEE Transactions on Neural Networks 1994; 5:240-254.

23. Feng JC, Tse CK, Lau FCM. A chaos tracker applied to non-coherent detection in chaos-based digital communication systems. Proceedings of IEEE ISCAS 2001; 3:795-798.

24. Chua LO, Komuro M, Matsumoto T. The double scroll family. IEEE Transactions on Circuits and Systems 1986; 33:1073-1117.

25. Chialina S, Hasler M, Prempli A. Fast and accurate calculation of Lyapunov exponents for piece-wise linear systems, International Journal of Bifurcation and Chaos 1994; 4:127-136.

26. Khalid AA, Irving SR. The use of neural nets to combine equalization with decoding for severe intersymbol interference channels. IEEE Transactions on Neural Networks 1994; 5:982-988.

27. Chen S, Mulgrew B, Grant PM. A clustering teaching for digital communications channel equalization using radial basis function networks. IEEE Transactions on Neural Networks 1993; 4:570-590.

28. Takens F. Detecting strange attractors in turbulence. In Dynamical Systems and Turbulence, Rand D, Young I (eds). Springer: Berlin, 1981.

29. Gencay R, Liu T. Nonlinear modelling and prediction with feedforward and recurrent networks. Physica D 1997; 108:119-134

30. Haykin S. Neural Networks: A Comprehensive Foundation. Prentice-Hall Inc.: Englewood Cliffs, NJ, 1994.

\section{AUTHORS' BIOGRAPHIES}

Jiuchao Feng received the BS degree in physics from Southwest China Normal University, in 1986, the ME degree in communication and electronic systems from South China University of technology, Guangzhou, China, in 1997, and the PhD degree from Hong Kong Polytechnic University, Hong Kong, in 2002. He is with School of Electronic and Information Engineering, South China University of Technology, Guangzhou 510641, China. His areas of interests include digital signal processing, communication theory, non-linear dynamics and chaos.

Chi K. Tse received the BEng degree (with first class honors) in electrical engineering and the $\mathrm{PhD}$ degree from the University of Melbourne, Australia, in 1987 and 1991, respectively. He is presently a Professor 
with Hong Kong Polytechnic University, Hong Kong, and his research interests include chaotic dynamics and power electronics. He is the author of Linear Circuit Analysis (London, U.K.: Addison-Wesley, 1998), co-author of Chaos-Based Digital Communication Systems (Heidelberg, Germany: Springer-Verlag, 2003), and co-holder of a U.S. patent. Since 2002, he has been appointed as Advisory Professor by the Southwest China Normal University, Chongqing, China. Dr. Tse received the L.R. East Prize by the Institution of Engineers, Australia, in 1987, the IEEE Transactions on Power Electronics Prize Paper Award, in 2001, the President's Award for Achievement in Research, twice, and the Faculty's Best Researcher Award. He was an Associate Editor for the IEEE Transactions on Circuits and Systems Part I-Fundamental Theory and Applications, from 1999 to 2001, and since 1999 he has been an Associate Editor for the IEEE Transactions on Power Electronics.

Francis C. M. Lau received the BEng (Hons) degree with first class honors in electrical and electronic engineering and the PhD degree from King's College London, University of London, U.K., in 1989 and 1993, respectively. He is now an Associate Professor at Department of Electronic and Information Engineering, the Hong Kong Polytechnic University, Hong Kong. His main research interests include power control and capacity analysis in mobile communication systems and chaos-based digital communications. 